\title{
Limit Cycles and Isochronous Centers in a Class of Ninth Degree System
}

\author{
Li Hongwei, ${ }^{1}$ Li Feng, ${ }^{1}$ and Du Chaoxiong ${ }^{2}$ \\ ${ }^{1}$ School of Science, Linyi University, Linyi, Shandong 276005, China \\ ${ }^{2}$ School of Science, Shaoyang University, Shaoyang, Hunan 422000, China
}

Correspondence should be addressed to Li Feng; lf0539@126.com

Received 15 April 2013; Accepted 3 October 2013

Academic Editor: Ruediger Landes

Copyright (C) $2013 \mathrm{Li}$ Hongwei et al. This is an open access article distributed under the Creative Commons Attribution License, which permits unrestricted use, distribution, and reproduction in any medium, provided the original work is properly cited.

A class of ninth degree system is studied and the conditions ensuring that its five singular points can be centers and isochronous centers (or linearizable centers) at the same time by exact calculation and strict proof are obtained. What is more, the expressions of Lyapunov constants and periodic constants are simplified, and 21 limit circles could be bifurcated at least.

\section{Introduction}

As we all know, it is difficult for planar polynomial differential systems to characterize their centers and isochronous centers. Hence, up to now the sufficient and necessary condition determining an isochronous center can only be found by making some appropriate analytic changes of coordinates which let the original system be reduced to a linear system. This kind of appropriate analytic change is very difficult to obtain, so only a handful of isochronous systems have been investigated. Several classes of known isochronous systems studied are as follows: quadratic isochronous centers (see [1]); isochronous centers of a linear center perturbed by third, fourth and fifth degree homogeneous polynomials (see [24]); complex polynomial systems (see [5]); reversible systems (see $[6,7])$; and isochronous centers of cubic systems with degenerate infinity (see [8]).

In [9], a class of ninth degree system with four isochronous centers was investigated. In this paper, we consider the following ninth degree system:

$$
\begin{aligned}
\frac{d x}{d t}= & -\lambda y\left(x^{2}+y^{2}\right)^{4} \\
& +y\left(p_{40} x^{4}+p_{31} x^{3} y+p_{22} x^{2} y^{2}+p_{04} y^{4}\right) \\
& +y\left(p_{60} x^{6}+p_{42} x^{4} y^{2}+p_{24} x^{2} y^{4}+p_{06} y^{6}\right)
\end{aligned}
$$

$$
\begin{aligned}
\frac{d y}{d t}= & \lambda x\left(x^{2}+y^{2}\right)^{4} \\
& +x\left(q_{40} x^{4}+q_{22} x^{2} y^{2}+q_{13} x y^{3}+q_{04} y^{4}\right) \\
& +x\left(q_{60} x^{6}+q_{42} x^{4} y^{2}+q_{24} x^{2} y^{4}+q_{06} y^{6}\right),
\end{aligned}
$$

where

$$
\begin{gathered}
p_{40}=-q_{04}=\frac{1}{2}\left(-10 \lambda+4 A_{32}+8 A_{50}-5 \beta\right), \\
p_{31}=-q_{13}=-8 B_{50}, \\
p_{22}=-q_{22}=10 \lambda-6 A_{32}-4 A_{50}+5 \beta, \\
p_{04}=-q_{40}=\frac{1}{2}(-2 \lambda-\beta), \\
p_{60}=-q_{06}=\frac{1}{2}\left(12 \lambda-4 A_{32}-8 A_{50}+7 \beta\right), \\
p_{42}=-q_{24}=\frac{1}{2}\left(-4 \lambda+8 A_{32}+16 A_{50}-9 \beta\right), \\
p_{24}=-q_{42}=-\frac{3}{2}\left(4 \lambda-4 A_{32}-8 A_{50}+5 \beta\right), \\
p_{06}=-q_{60}=\frac{1}{2}(4 \lambda+\beta), \quad \lambda \beta \neq 0 .
\end{gathered}
$$


The paper is organized as follows. In Section 2, the preliminary methods to calculate the focal values (or Lyapunov constants) and period constants which are necessary in Sections 3 and 5 are presented. In Section 3, two appropriate transformations, changing system (1) to a class of Z4equivariant fifth degree system in which the focal values are calculated, are provided. Based on it, the condition and proof that the infinity and four elementary singular points of (1) can be centers are gained. The bifurcations of 21 limit circles are the subject of Section 4. At last, in Section 5, the period constants with simpler expressions are given; moreover, the sufficient and necessary condition and proof are given to illustrate that the infinity and four elementary singular points of (1) become isochronous centers.

\section{Some Preliminary Results}

In $[10,11]$, the complex system of complex center is

$$
\begin{gathered}
\frac{d z}{d T}=z+\sum_{k=2}^{\infty} Z_{k}(z, w)=Z(z, w), \\
\frac{d w}{d T}=-w-\sum_{k=2}^{\infty} W_{k}(z, w)=-W(z, w),
\end{gathered}
$$

where

$$
Z_{k}(z, w)=\sum_{\alpha+\beta=k} a_{\alpha \beta} z^{\alpha} w^{\beta}, \quad W_{k}(z, w)=\sum_{\alpha+\beta=k} b_{\alpha \beta} w^{\alpha} z^{\beta},
$$

and the authors gave two recursive algorithms to determine necessary conditions for a center. We now restate the definitions and algorithms.

Theorem 1 (see [11]). For system (3), we can derive successively the terms of the following formal series:

$$
M(z, w)=\sum_{\alpha+\beta=0}^{\infty} c_{\alpha \beta} z^{\alpha} w^{\beta}
$$

such that

$$
\frac{\partial(M Z)}{\partial z}-\frac{\partial(M W)}{\partial w}=\sum_{m=1}^{\infty}(m+1) \mu_{m}(z w)^{m}
$$

where $c_{00}=1$, for all $c_{k k} \in R, k=1,2, \ldots$, and for any integer $m, \mu_{m}$ is determined by the following recursive formulae:

$c_{00}=1$;

when $(\alpha=\beta>0)$ or $\alpha<0$, or $\beta<0, c_{\alpha \beta}=0$;

else

$$
\begin{aligned}
& c_{\alpha \beta}=\frac{1}{\beta-\alpha} \sum_{k+j=3}^{\alpha+\beta+2}\left[(\alpha+1) a_{k, j-1}-(\beta+1) b_{j, k-1}\right] c_{\alpha-k+1, \beta-j+1}, \\
& \mu_{m}=\sum_{k+j=3}^{2 m+2}\left(a_{k, j-1}-b_{j, k-1}\right) c_{m-k+1, m-j+1} .
\end{aligned}
$$

Theorem 2 (see [12]). For system (3), we can derive uniquely the following formal series:

$$
\begin{aligned}
& f(z, w)=z+\sum_{k+j=2}^{\infty} c_{k j}^{\prime} z^{k} w^{j}, \\
& g(z, w)=w+\sum_{k+j=2}^{\infty} d_{k j}^{\prime} w^{k} z^{j},
\end{aligned}
$$

where $c_{k+1, k}^{\prime}=d_{k+1, k}^{\prime}=0, k=1,2, \ldots$, such that

$$
\begin{aligned}
& \frac{d f}{d T}=f(z, w)+\sum_{j=1}^{\infty} p_{j}^{\prime} z^{j+1} w^{j}, \\
& \frac{d g}{d T}=-g(z, w)-\sum_{j=1}^{\infty} q_{j}^{\prime} w^{j+1} z^{j},
\end{aligned}
$$

and when $k-j-1 \neq 0, c_{k j}^{\prime}$ and $d_{k j}^{\prime}$ are determined by the following recursive formulae:

$$
\begin{aligned}
& c_{k j}^{\prime}=\frac{1}{j+1-k} \sum_{\alpha+\beta=3}^{k+j+1}\left[(k-\alpha+1) a_{\alpha, \beta-1}\right. \\
& \left.\quad-(j-\beta+1) b_{\beta, \alpha-1}\right] c_{k-\alpha+1, j-\beta+1}^{\prime}, \\
& d_{k j}^{\prime}=\frac{1}{j+1-k} \sum_{\alpha+\beta=3}^{k+j+1}\left[(k-\alpha+1) b_{\alpha, \beta-1}\right. \\
& \left.\quad-(j-\beta+1) a_{\beta, \alpha-1}\right] d_{k-\alpha+1, j-\beta+1}^{\prime},
\end{aligned}
$$

and for any positive integer $j, p_{j}^{\prime}$ and $q_{j}^{\prime}$ are determined by the following recursive formulae:

$$
\begin{aligned}
& p_{j}^{\prime}=\sum_{\alpha+\beta=3}^{2 j+2}[(j-\alpha+2) a_{\alpha, \beta-1} \\
&\left.-(j-\beta+1) b_{\beta, \alpha-1}\right] c_{j-\alpha+2, j-\beta+1}^{\prime}, \\
& q_{j}^{\prime}=\sum_{\alpha+\beta=3}^{2 j+2}\left[(j-\alpha+2) b_{\alpha, \beta-1}\right. \\
&\left.\quad-(j-\beta+1) a_{\beta, \alpha-1}\right] d_{j-\alpha+2, j-\beta+1}^{\prime} .
\end{aligned}
$$

In the above expression, we have let $c_{10}^{\prime}=d_{10}^{\prime}=1, c_{01}^{\prime}=d_{01}^{\prime}=$ 0 , and if $\alpha<0$ or $\beta<0$, let $a_{\alpha \beta}=b_{\alpha \beta}=c_{\alpha \beta}^{\prime}=d_{\alpha \beta}^{\prime}=0$.

\section{Singular Point Quantities and Center Conditions}

It can be easily checked that system (1) have four elementary critical points $( \pm 1,0),(0, \pm 1)$ and the infinity. After introducing the method to calculate the focal values and period constants of system (1), we try to make some appropriate transformations so as to carry out our investigation. 

tion

By means of the Bendixson homeomorphous transforma-

$$
u=\frac{x}{x^{2}+y^{2}}, \quad v=\frac{y}{x^{2}+y^{2}}
$$

and time transformation

$$
d t=\left(x^{2}+y^{2}\right)^{4} d \tau
$$

system (1) can be transformed into the following real system:

$$
\begin{aligned}
\frac{d u}{d \tau}= & -\lambda v+\left(-2 \lambda+2 A_{32}+4 A_{50}-\frac{5}{2} \beta\right) u^{2} v \\
& +\left(2 \lambda+\frac{\beta}{2}\right) v^{3}+\left(3 \lambda-2 A_{32}-4 A_{50}+\frac{3}{2} \beta\right) u^{4} v \\
& +8 B_{50} u^{3} v^{2}+\left(2 \lambda-2 A_{32}+4 A_{50}+\beta\right) u^{2} v^{3} \\
& -\left(\lambda+\frac{\beta}{2}\right) v^{5}, \\
\frac{d v}{d \tau}= & \lambda u-\left(2 \lambda+\frac{\beta}{2}\right) u^{3}+\left(2 \lambda-2 A_{32}-4 A_{50}+\frac{5}{2} \beta\right) u v^{2} \\
& +\left(\lambda+\frac{\beta}{2}\right) u^{5}+\left(-2 \lambda+2 A_{32}-4 A_{50}-\beta\right) u^{3} v^{2} \\
& -8 B_{50} u^{2} v^{3}+\left(-3 \lambda+2 A_{32}+4 A_{50}-\frac{3}{2} \beta\right) u v^{4} .
\end{aligned}
$$

Then, the infinity and four elementary focuses $( \pm 1,0),(0, \pm 1)$ of (1) become the origin and $( \pm 1,0),(0, \pm 1)$ of system $(14)$, respectively. Thus, we have the following theorems.

Theorem 3. System (14) is a class of Z4-equivariant differential polynomial system of fifth degree about point $(-1,0)$.

Proof. The system is invariant by the transformation of rotation

$$
u=x \cos \frac{\pi}{2}-y \sin \frac{\pi}{2}, \quad v=x \cos \frac{\pi}{2}+y \sin \frac{\pi}{2} .
$$

Then system (14) is a Z4-equivariant polynomial system about point $(-1,0)$.

Now, we will consider the $(0,0)$ of system $(14)$. Let

$$
z=x+i y, \quad w=x-i y, \quad T=i t .
$$

System (14) can be transformed into the following complex system:

$$
\begin{aligned}
\frac{d z}{d T}= & \lambda z-\left(\lambda+a_{32}+2 a_{50}+\frac{1}{4} \beta\right) w z^{2} \\
& +\left(a_{32}-\lambda+2 a_{50}-\frac{3 \beta}{4}\right) w^{3}+a_{50} z^{5} \\
& +\left(\lambda-a_{32}-a_{50}+\frac{1}{2} \beta\right) w^{4} z+a_{32} w^{2} z^{3}
\end{aligned}
$$

$$
\begin{aligned}
\frac{d w}{d T}= & -\lambda w+\left(\lambda+b_{32}+2 b_{50}+\frac{1}{4} \beta\right) z w^{2} \\
& -\left(b_{32}-\lambda+2 b_{50}-\frac{3 \beta}{4}\right) z^{3}-b_{50} w^{5} \\
& -\left(\lambda-b_{32}-b_{50}+\frac{1}{2} \beta\right) z^{4} w-b_{32} z^{2} w^{3},
\end{aligned}
$$

where

$$
\begin{aligned}
a_{50}=\frac{A_{50}+B_{50} i}{2}, & b_{50}=\frac{A_{50}-B_{50} i}{2}, \\
a_{32}=\frac{A_{32}-2 B_{50} i}{2}, & b_{32}=\frac{A_{32}+2 B_{50} i}{2} .
\end{aligned}
$$

Theorem 4. The first two singular point quantities at the origin of system (17) are as follows:

$$
u_{1}=0, \quad u_{2}=\frac{2 i B_{50}}{\lambda} .
$$

Theorem 5. For system (17), the first two singular point quantities are zero if and only if the following condition holds:

$$
B_{50}=0 .
$$

Theorem 6. For system (14), all the singular point quantities at the origin are zero if and only if the first two singular point quantities are zero; that is, the condition in Theorem 5 holds. Correspondingly, the condition in Theorem 5 is the center condition of the origin.

Proof. If condition (20) holds, system (14) becomes

$$
\begin{aligned}
\frac{d u}{d \tau}= & -\lambda v+\left(-2 \lambda+2 A_{32}+4 A_{50}-\frac{5}{2} \beta\right) u^{2} v \\
& +\left(2 \lambda+\frac{\beta}{2}\right) v^{3}+\left(3 \lambda-2 A_{32}-4 A_{50}+\frac{3}{2} \beta\right) u^{4} v \\
& +\left(2 \lambda-2 A_{32}+4 A_{50}+\beta\right) u^{2} v^{3}-\left(\lambda+\frac{\beta}{2}\right) v^{5} \\
\frac{d v}{d \tau}= & \lambda u-\left(2 \lambda+\frac{\beta}{2}\right) u^{3}+\left(2 \lambda-2 A_{32}-4 A_{50}+\frac{5}{2} \beta\right) u v^{2} \\
& +\left(\lambda+\frac{\beta}{2}\right) u^{5}+\left(-2 \lambda+2 A_{32}-4 A_{50}-\beta\right) u^{3} v^{2} \\
& +\left(-3 \lambda+2 A_{32}+4 A_{50}-\frac{3}{2} \beta\right) u v^{4} .
\end{aligned}
$$

Remark (21) is symmetric with $v$ axis.

System (14) is a class of Z4-equivariant differential polynomial system, so that the four symmetric elementary focus points of system (14) have the same topological structure; then they have the same center conditions and isochronous center conditions and so on. Without loss of generality, we only need to consider the case of $(1,0)$. 
Let

$$
x=u-1, \quad y=v .
$$

Then, system (14) can be changed into

$$
\begin{aligned}
& \frac{d x}{d t}=-\beta y+\left(8 \lambda-4 A_{32}-8 A_{50}+\beta\right) x y \\
& +\left(16 \lambda-10 A_{32}-20 A_{50}+\frac{13}{2} \beta\right) x^{2} y \\
& +\left(12 \lambda-8 A_{32}-16 A_{50}+6 \beta\right) x^{3} y \\
& +\left(3 \lambda-2 A_{32}-4 A_{50}+\frac{3}{2} \beta\right) x^{4} y+8 B_{50} y^{2} \\
& +8 B_{50} x^{3} y^{2}+\left(4 \lambda-2 A_{32}+4 A_{50}+\frac{3}{2} \beta\right) y^{3} \\
& +\left(4 \lambda-4 A_{32}+8 A_{50}+2 \beta\right) x y^{3}+24 B_{50} x y^{2} \\
& +24 B_{50} x^{2} y^{2}+\left(2 \lambda-2 A_{32}+4 A_{50}+\beta\right) x^{2} y^{3} \\
& -\left(\lambda+\frac{1}{2} \beta\right) y^{5} \\
& \frac{d y}{d t}=\beta x+\left(4 \lambda+\frac{7}{2} \beta\right) x^{2}+\left(8 \lambda+\frac{9}{2} \beta\right) x^{3} \\
& +\left(5 \lambda+\frac{5}{2} \beta\right) x^{4}+\left(\lambda+\frac{1}{2} \beta\right) x^{5}+\left(\frac{3}{2} \beta-8 A_{50}\right) y^{2} \\
& +\left(4 A_{32}-4 \lambda-16 A_{50}-\frac{1}{2} \beta\right) x y^{2} \\
& +\left(6 A_{32}-6 \lambda-12 A_{50}-3 \beta\right) x^{2} y^{2} \\
& +\left(-2 \lambda+2 A_{32}-4 A_{50}-\beta\right) x^{3} y^{2} \\
& +8 B_{50} y^{3}+16 B_{50} x y^{3}+8 B_{50} x^{2} y^{3} \\
& +\left(-3 \lambda+2 A_{32}+4 A_{50}-\frac{3}{2} \beta\right) y^{4} \\
& +\left(-3 \lambda+2 A_{32}+4 A_{50}-\frac{3}{2} \beta\right) x y^{4} .
\end{aligned}
$$

By noting that

$$
z=x+i y, \quad w=x-i y, \quad T=i t
$$

system (23) can be transformed into its complex system:

$$
\begin{aligned}
\frac{d z}{d T}= & \beta z+\left(3 \lambda-2 a_{32}+\frac{3}{4} \beta\right) w^{2} \\
& +\left(3 \lambda-3 a_{32}-2 a_{50}+\frac{5}{4} \beta\right) w^{3} \\
& +\left(\lambda-a_{32}-a_{50}+\frac{1}{2} \beta\right) w^{4} \\
& +\left(2 \lambda-8 a_{50}+\frac{5}{2} \beta\right) w z \\
& +\left(6 \lambda-3 a_{32}-6 a_{50}+3 \beta\right) w^{2} z
\end{aligned}
$$

$$
\begin{aligned}
& +\left(4 \lambda-4 a_{32}-4 a_{50}+2 \beta\right) w^{3} z \\
& +\left(\lambda-a_{32}-a_{50}+\frac{1}{2} \beta\right) w^{4} z \\
& +\left(2 a_{32}-\lambda+8 a_{50}+\frac{1}{4} \beta\right) z^{2} \\
& +\left(5 a_{32}-\lambda-2 a_{50}+\frac{1}{4} \beta\right) w z^{2} \\
& +3 a_{32} w^{2} z^{2}+\left(a_{32}+10 a_{50}\right) z^{3}+2 a_{32} w z^{3} \\
& +a_{32} w^{2} z^{3}+5 a_{50} z^{4}+a_{50} z^{5} \\
& \frac{d w}{d T}=-\beta w-\left(3 \lambda-2 b_{32}+\frac{3}{4} \beta\right) z^{2} \\
& -\left(3 \lambda-3 b_{32}-2 b_{50}+\frac{5}{4} \beta\right) z^{3} \\
& -\left(\lambda-b_{32}-b_{50}+\frac{1}{2} \beta\right) z^{4} \\
& -\left(2 \lambda-8 b_{50}+\frac{5}{2} \beta\right) w z \\
& -\left(6 \lambda-3 b_{32}-6 b_{50}+3 \beta\right) z^{2} w \\
& -\left(4 \lambda-4 b_{32}-4 b_{50}+2 \beta\right) z^{3} w \\
& -\left(\lambda-b_{32}-b_{50}+\frac{1}{2} \beta\right) z^{4} w \\
& -\left(2 b_{32}-\lambda+8 b_{50}+\frac{1}{4} \beta\right) w^{2} \\
& -\left(5 b_{32}-\lambda-2 b_{50}+\frac{1}{4} \beta\right) z w^{2} \\
& -3 b_{32} w^{2} z^{2}-\left(b_{32}+10 b_{50}\right) w^{3}-2 b_{32} z w^{3} \\
& -b_{32} z^{2} w^{3}-5 b_{50} w^{4}-b_{50} w^{5} \text {. }
\end{aligned}
$$

Through computing, we have the following theorem.

Theorem 7. The first five singular point quantities at the origin of system (25) are

$$
\begin{gathered}
u_{1}=-\frac{4 i B_{50}\left(4 \lambda-2 A_{32}-12 A_{50}+5 \beta\right)}{\beta^{2}}, \\
u_{2}=\frac{8 i B_{50}\left(\beta-4 A_{50}\right)\left(2 A_{32}-28 A_{50}+5 \beta\right)}{\beta^{3}}, \\
u_{3}=8 i B_{50}\left(\beta-4 A_{50}\right)^{2}\left(-4480 A_{50}^{2}+560 B_{50}^{2}\right. \\
\left.+1992 A_{50} \beta-215 \beta^{2}\right)\left(\beta^{5}\right)^{-1} \\
u_{4}=-\frac{64 i B_{50}\left(\beta-4 A_{50}\right)^{2} f}{175 \beta^{7}} \\
u_{5}=-\frac{16384 i A_{50}^{3} B_{50}\left(\beta-4 A_{50}\right)^{2} g}{1827307548828125 \beta^{9}}
\end{gathered}
$$


where

$$
\begin{aligned}
f= & 67737600 A_{50}^{4}-63598080 A_{50}^{3} \beta+22017616 A_{50}^{2} \beta^{2} \\
& -3335040 A_{50} \beta^{3}+186875 \beta^{4} \\
g= & 16146722095087449600 A_{50}^{3} \\
& -9886197819204477680 A_{50}^{2} \beta \\
& +2031858784197168536 A_{50} \beta^{2} \\
& -142685023001803215 \beta^{3} .
\end{aligned}
$$

Theorem 8. For system (25), the first five singular point quantities are zero if and only if one of the following conditions holds:

$$
\begin{gathered}
\lambda=\frac{1}{2}\left(A_{32}-4 A_{50}\right), \quad \beta=4 A_{50} ; \\
B_{50}=0 .
\end{gathered}
$$

Then we can get the following theorem.

Theorem 9. For system (25), all the singular point quantities at the origin are zero if and only if the first five singular point quantities are zero; that is, one of the conditions in Theorem 8 holds. Correspondingly, the conditions in Theorem 8 are the center conditions of the origin.

Proof. Under condition (28), the system (25) becomes

$$
\begin{aligned}
\frac{d x}{d t}= & -4 A_{50} y-20 A_{50} x y-\left(2 A_{32}+26 A_{50}\right) x^{2} y \\
& -\left(2 A_{32}+16 A_{50}\right) x^{3} y-\left(\frac{1}{2} A_{32}+4 A_{50}\right) x^{4} y \\
& +8 B_{50} y^{2}+24 B_{50} x y^{2}+24 B_{50} x^{2} y^{2} \\
& +8 B_{50} x^{3} y^{2}+2 A_{50} y^{3}+\left(8 A_{50}-2 A_{32}\right) x y^{3} \\
& +\left(4 A_{50}-A_{32}\right) x^{2} y^{3}-\frac{1}{2} A_{32} y^{5}, \\
\frac{d y}{d t}= & 4 A_{50} x+2\left(A_{32}+3 A_{50}\right) x^{2}+2\left(2 A_{32}+A_{50}\right) x^{3} \\
& +\frac{5}{2} A_{32} x^{4}+\frac{1}{2} A_{32} x^{5}-2 A_{50} y^{2}+2\left(A_{32}-5 A_{50}\right) x y^{2} \\
& +3\left(A_{32}-4 A_{50}\right) x^{2} y^{2}+\left(A_{32}-4 A_{50}\right) x^{3} y^{2} \\
& +8 B_{50} y^{3}+16 B_{50} x y^{3}+8 B_{50} x^{2} y^{3} \\
& +\left(\frac{1}{2} A_{32}+4 A_{50}\right) y^{4}+\left(\frac{1}{2} A_{32}+4 A_{50}\right) x y^{4} . \\
& +30)(30)
\end{aligned}
$$

System (30) has an integrating factor

$$
u(x, y)=\frac{1}{\left(1+2 x+x^{2}+y^{2}\right)^{3}}
$$

Under condition (29), system (25) becomes

$$
\begin{aligned}
& \frac{d x}{d t}=-\beta y+\left(8 \lambda-4 A_{32}-8 A_{50}+\beta\right) x y \\
& +\left(16 \lambda-10 A_{32}-20 A_{50}+\frac{13}{2} \beta\right) x^{2} y \\
& +\left(12 \lambda-8 A_{32}-16 A_{50}+6 \beta\right) x^{3} y \\
& +\left(3 \lambda-2 A_{32}-4 A_{50}+\frac{3}{2} \beta\right) x^{4} y \\
& +\left(4 \lambda-2 A_{32}+4 A_{50}+\frac{3}{2} \beta\right) y^{3} \\
& +\left(4 \lambda-4 A_{32}+8 A_{50}+2 \beta\right) x y^{3} \\
& +\left(2 \lambda-2 A_{32}+4 A_{50}+\beta\right) x^{2} y^{3}-\left(\lambda+\frac{1}{2} \beta\right) y^{5}, \\
& \frac{d y}{d t}=\beta x+\left(4 \lambda+\frac{7}{2} \beta\right) x^{2}+\left(8 \lambda+\frac{9}{2}\right) \beta x^{3} \\
& +\left(5 \lambda+\frac{5}{2} \beta\right) x^{4}+\left(\lambda+\frac{1}{2} \beta\right) x^{5}+\left(-8 A_{50}+\frac{3}{2} \beta\right) y^{2} \\
& +\left(-4 \lambda+4 A_{32}-16 A_{50}-\frac{1}{2} \beta\right) x y^{2} \\
& +\left(-6 \lambda+6 A_{32}-12 A_{50}-3 \beta\right) x^{2} y^{2} \\
& +\left(-2 \lambda+2 A_{32}-4 A_{50}-\beta\right) x^{3} y^{2} \\
& +\left(-3 \lambda+2 A_{32}+4 A_{50}-\frac{3}{2} \beta\right) y^{4} \\
& +\left(-3 \lambda+2 A_{32}+4 A_{50}-\frac{3}{2} \beta\right) x y^{4},
\end{aligned}
$$

where (32) is symmetric with $x$ axis.

So we get the following theorem.

Theorem 10. The system (14) has five centers $(0,0),( \pm 1,0)$, $(0, \pm 1)$ when $B_{50}=0$. Namely, the system (1) has five centers the infinity and $( \pm 1,0),(0, \pm 1)$ when $B_{50}=0$.

The system (14) has four centers $( \pm 1,0),(0, \pm 1)$ when $\lambda=$ $(1 / 2)\left(A_{32}-4 A_{50}\right), \beta=4 A_{50}, B_{50} \neq 0$. Namely, the system (1) has four centers $( \pm 1,0),(0, \pm 1)$ when $\lambda=(1 / 2)\left(A_{32}-4 A_{50}\right)$, $\beta=4 A_{50}$, and $B_{50} \neq 0$. 


\section{Bifurcation of Limit Circles}

Theorem 11. For system (14), the $(1,0)$ is a fifth order weak focus if and only if

$$
\begin{gathered}
\lambda=\frac{1}{2}\left(20 A_{50}-5 \beta\right), \\
A_{32}=\frac{1}{2}\left(28 A_{50}-5 \beta\right),
\end{gathered}
$$

$$
B_{50}^{2}>0, \quad u_{4}=0
$$

Resultant $\left[f, g, A_{50}\right]$

$$
=16309978866916163079012825599795663295988359261138540000000000000000000000000000000000 \beta^{12} .
$$

So the $(1,0)$ is a fifth order weak focus.

Theorem 12. Assume that the conditions in Theorem 11 hold. Then the 5 limit cycles can bifurcate from the $(1,0)$ of System (14), which lie in the neighborhood of the origin.

Proof. If the conditions in Theorem 11 hold, we can obtain that the Jacobian of the $u_{1}, u_{2}, u_{3}, u_{4}$ with respect to $\lambda, A_{32}, B_{50}, A_{50}$

$$
\begin{aligned}
& J=\frac{D\left(u_{1}, u_{2}, u_{3}, u_{4}\right)}{D\left(\lambda, A_{32}, B_{50}, A_{50}\right)} \\
& =-\frac{2097152 B_{50}^{5}\left(-4 A_{50}+\beta\right)^{4}}{175 \beta^{1} 7} \\
& \quad \times\left(203212800 A_{50}^{4}-192864000 A_{50}^{3} \beta\right. \\
& \quad+67884512 A_{50} \beta^{2}-10506964 A_{50} \beta^{3} \\
& \left.\quad+603755 \beta^{4}\right) ; \\
& \text { Resultant }\left[f, J, A_{50}\right] \\
& =-661345270173473293422286219719475200000000 \beta^{16} .
\end{aligned}
$$

So $J \neq 0$. Applying the theory in [13], the result is completed.

Theorem 13. Assume that the conditions in Theorem 11 hold. Then 1 limit cycle can bifurcate from the $(0,0)$ of System (14), which lie in the neighborhood of the origin.

Since System (14) is Z4-equivariant, taking into account Theorems 12 and 13, we conclude the following.

Theorem 14. Assume that the conditions in Theorem 11 hold. Then 5 limit cycles can bifurcate from each of the four fine focus points $( \pm 1,0),(0, \pm 1)$ of System $(14)$, and one limit circle could be created from the origin at the same time; namely, 21 limit cycles can bifurcate from the System (14) in all, five of which are located in the neighborhood of each of the four fine focus points
$( \pm 1,0),(0, \pm 1)$ and one of which is located in the neighborhood of origin.

In fact, there are 21 limit cycles for system (1); 5 limit cycles can bifurcate from each of the four fine focus points $( \pm 1,0)$, $(0, \pm 1)$ of System $(1)$, and one limit circle could be created from the infinity at the same time; namely, 21 limit cycles can bifurcate from the system (1) in all. It is a very interesting result.

\section{Period Constants and Isochronous Center Conditions}

In this section, we focus on the calculation of period constants and isochronous center conditions of the origin and $(1,0)$ for system (17) and (25). According to Theorems 2, we begin by computing period constants through recursive formulae. Under the center conditions given in Section 3, we discuss three different relevant cases: (a) about the origin, case condition (20) holds.

Theorem 15. Under condition (20), the origin of system (17) is a complex isochronous center if and only if the following conditions holds

$$
A_{32}=B_{50}=0, \quad A_{50}=-2 \lambda, \quad \beta=-4 \lambda .
$$

Proof. If condition (20) holds, substituting $B_{50}=0$ into the recursive formulae in Theorem 2 , we can easily get the first four period constants

$$
\begin{gathered}
\tau_{1}=-\frac{4 \lambda+2 A_{32}+4 A_{50}-\beta}{2 \lambda}, \\
\tau_{2}=-\frac{64 \lambda^{2}+16 \lambda A_{50}+20 \lambda \beta+3 \beta^{2}}{8 \lambda^{2}}, \\
\tau_{3}=-\frac{(4 \lambda+\beta)\left(80 \lambda^{2}+46 \lambda \beta+9 \beta^{2}\right)}{32 \lambda^{3}}, \\
\tau_{4}=\frac{3 \beta(4 \lambda+\beta)\left(104 \lambda^{2}+40 \lambda \beta+9 \beta^{2}\right)}{512 \lambda^{4}} .
\end{gathered}
$$


Clearly, $\tau_{1}=\tau_{2}=\tau_{3}=\tau_{4}=0$ implies that condition (37) holds.

On the other hand, case condition (37) holds, and system (17) is changed into

$$
\begin{gathered}
\frac{d z}{d T}=-z(z+1)(z-1)\left(z^{2}+1\right), \\
\frac{d w}{d T}=(-1+w) w(1+w)\left(1+w^{2}\right) .
\end{gathered}
$$

By means of transformation

$$
\xi=z\left(z^{4}-1\right)^{-1 / 4}, \quad \eta=w\left(w^{4}-1\right)^{-1 / 4},
$$

system (39) is reduced to a linear system.

Thus, the origin of system (17) is a complex isochronous center.

(b) about $(1,0)$ of system $(25)$.

When condition (28) holds, substituting $\lambda=(1 / 2)\left(A_{32}-\right.$ $\left.4 A_{50}\right), \beta=4 A_{50}$, into the recursive formulae in Theorem 2 , we easily obtain the first two period constants

$$
\tau_{1}=-\frac{A_{32}-2 A_{50}}{A_{32}-4 A_{50}}, \quad \tau_{2}=-2 .
$$

Thus $(1,0)$ could not be an isochronous center when condition (28) holds.

Theorem 16. $(1,0)$ of system $(25)$ is a complex isochronous center if and only if condition (37) holds.

Proof. When condition (29) holds, substituting $B_{50}$ into the recursive formulae in Theorem 2 , we easily obtain the first four period constants:

$$
\begin{gathered}
\tau_{1}=\frac{-4 \lambda+10 A_{32}-4 A_{50}+\beta}{2}, \\
\tau_{2}=\left(80 \lambda-13392 \lambda^{2}+80 A_{50}+17856 \lambda A_{50}\right. \\
-5952 A_{50}^{2}-20 \beta-15624 \lambda \beta \\
\left.+10416 A_{50} \beta-4557 \beta^{2}\right)(200)^{-1}, \\
\tau_{3}=\frac{1}{8000}\left(-237312 \lambda^{2}+2939328 \lambda^{3}\right. \\
+208896 \lambda A_{50}-5878656 \lambda^{2} A_{50} \\
-46592 A_{50}^{2}-870912 A_{50}^{3} \\
-299104 \lambda \beta+5143824 \lambda^{2} \beta \\
+162816 A_{50} \beta-6858432 A_{50} \lambda \beta \\
+2286144 A_{50}^{2} \beta-103592 \beta^{2}
\end{gathered}
$$

$$
\begin{aligned}
& +3000564 \lambda \beta^{2}-2000376 A_{50} \beta^{2} \\
& \left.+583443 \beta^{3}+3919104 \lambda A_{50}^{2}\right), \\
\tau_{4}=\frac{1}{40000}( & -51200 \lambda^{2}+28097664 \lambda^{3}+17356032 \lambda^{4} \\
+ & 57600 \lambda A_{50}-29498368 \lambda^{2} A_{50} \\
& -46282752 \lambda^{3} A_{50}+6545472 \lambda A_{50}^{2} \\
+ & 46282752 \lambda^{2} A_{50}^{2}+421504 A_{50}^{3} \\
& -20570112 \lambda A_{50}^{3}+3428352 A_{50}^{4} \\
& -70400 \lambda \beta+40046432 \lambda^{2} \beta \\
+ & 40497408 \lambda^{3} \beta+39600 A_{50} \beta \\
& -11200 A_{50}^{2}-80994816 \lambda^{2} A 50 \beta \\
+ & 996112 A_{50}^{2} \beta+53996544 \lambda A_{50}^{2} \beta \\
- & 11999232 A_{50}^{3} \beta-24200 \beta^{2} \\
+ & 17793272 \lambda \beta^{2}+35435232 \lambda^{2} \beta^{2} \\
- & 3856888 A_{50} \beta^{2}-47246976 \lambda A_{50} \beta^{2} \\
- & 24098656 \lambda A 50 \beta+15748992 A_{50}^{2} \beta^{2} \\
+ & 2329754 \beta^{3}+13780368 \lambda \beta^{3} \\
- & \left.9186912 A_{50} \beta^{3}+2009637 \beta^{4}\right) .
\end{aligned}
$$

Denoting by

$$
g_{1}=R\left(\tau_{2}, \tau_{3}, \lambda\right), \quad g_{2}=R\left(\tau_{2}, \tau_{4}, \lambda\right),
$$

where $R\left(\tau_{i}, \tau_{j}, A\right)$ is the resultant of $\tau_{i}, \tau_{j}$ with respect to $A$, we have $R\left(g_{1}, g_{2}, \beta\right)=0$. From polynomioalremain$\operatorname{der}\left[g_{2}, g_{1}, A_{50}\right]=0$, we could get that $\beta=2 A_{50}$. When $\beta=2 A_{50}, \tau_{2}=\tau_{3}=\tau_{4}=0$ deduce $A_{32}=B_{50}=0$, $A_{50}=-2 \lambda, \beta=-4 \lambda$.

Besides, when condition (37) holds, system (25) is changed into

$$
\begin{gathered}
\frac{d z}{d T}=\frac{1}{4} z(z+1)(z+2)\left(z^{2}+2 z+2\right), \\
\frac{d w}{d T}=-\frac{1}{4}(1+w) w(2+w)\left(2+2 w+w^{2}\right) .
\end{gathered}
$$

Letting that

$$
\begin{gathered}
\xi=\frac{z(2+z)\left(2+2 z+z^{2}\right)}{(z+1)^{4}}, \\
\eta=\frac{w(2+w)\left(2+2 w+w^{2}\right)}{(w+1)^{4}},
\end{gathered}
$$

system (44) is reduced to a linear system. 
From Theorems 15 and 16, we can conclude the following theorem.

Theorem 17. The infinity and four elementary singular points $( \pm 1,0),(0, \pm 1)$ are five isochronous centers of $(1)$ if and only if condition (37) holds; namely $A_{32}=B_{50}=0, A_{50}=-2 \lambda$, $\beta=-4 \lambda$.

\section{Acknowledgments}

This research is partially supported by the National Nature Science Foundation of China (11201211, 111101126, and 1371373) and Nature Science Foundation of Shandong Province (ZR2012AL04) and Applied Mathematics Enhancement Program of Linyi University.

\section{References}

[1] W. S. Loud, "Behavior of the period of solutions of certain plane autonomous systems near centers," vol. 3, pp. 21-36, 1964.

[2] J. Chavarriga, J. Giné, and I. A. García, "Isochronous centers of a linear center perturbed by fourth degree homogeneous polynomial," Bulletin des Sciences Mathématiques, vol. 123, no. 2, pp. 77-96, 1999.

[3] J. Chavarriga, J. Giné, and I. A. García, "Isochronous centers of a linear center perturbed by fifth degree homogeneous polynomials," Journal of Computational and Applied Mathematics, vol. 126, no. 1-2, pp. 351-368, 2000.

[4] I. Pleshkan, "A new method of investigating the isochronicity of system of two differential equations," Differential Equations, vol. 5, pp. 796-802, 1969.

[5] Y. P. Lin and J. B. Li, "Normal form and critical points values of the period of closed orbits for planar autonomous systems," Acta Mathematica Sinica, vol. 34, no. 4, pp. 490-501, 1991.

[6] J. Chavarriga, J. Giné, and I. A. García, "Isochronicity into a family of time-reversible cubic vector fields," Applied Mathematics and Computation, vol. 121, no. 2-3, pp. 129-145, 2001.

[7] L. Cairó, J. Giné, and J. Llibre, "A class of reversible cubic systems with an isochronous center," Computers \& Mathematics with Applications, vol. 38, no. 11-12, pp. 39-53, 1999.

[8] J. Chavarriga, J. Giné, and I. García, "Isochronous centers of cubic systems with degenerate infinity," Differential Equations and Dynamical Systems for Theory, Applications, and Computer Simulations, vol. 7, no. 2, pp. 221-238, 1999.

[9] C. Du, Y. Liu, and H. Mi, "A class of ninth degree system with four isochronous centers," Computers \& Mathematics with Applications, vol. 56, no. 10, pp. 2609-2620, 2008.

[10] Y. R. Liu and H. B. Chen, "Formulas of singular point quantities and the first 10 saddle quantities for a class of cubic system," Acta Mathematicae Applicatae Sinica, vol. 25, no. 2, pp. 295-302, 2002 (Chinese).

[11] Y. Liu and J. Li, "Theory of values of singular point in complex autonomous differential system," Science China. Series A, vol. 3, pp. 245-255, 1989.

[12] Y. Liu and W. Huang, "A new method to determine isochronous center conditions for polynomial differential systems," Bulletin des Sciences Mathématiques, vol. 127, no. 2, pp. 133-148, 2003.

[13] M. Han, Y. Lin, and P. Yu, "A study on the existence of limit cycles of a planar system with third-degree polynomials," International Journal of Bifurcation and Chaos in Applied Sciences and Engineering, vol. 14, no. 1, pp. 41-60, 2004. 


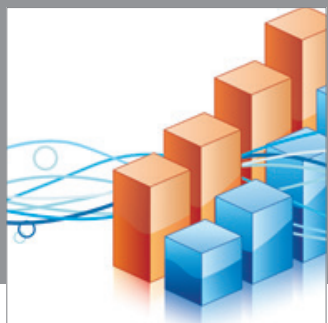

Advances in

Operations Research

mansans

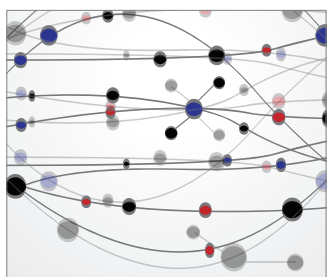

The Scientific World Journal
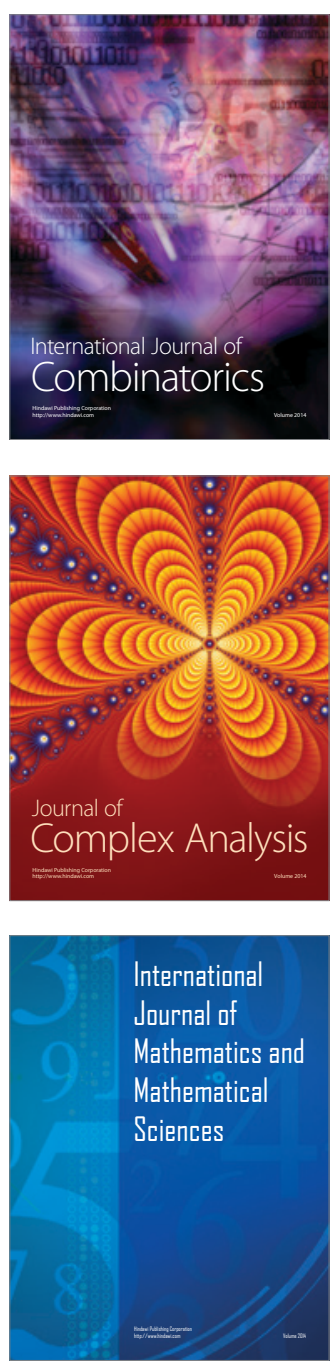
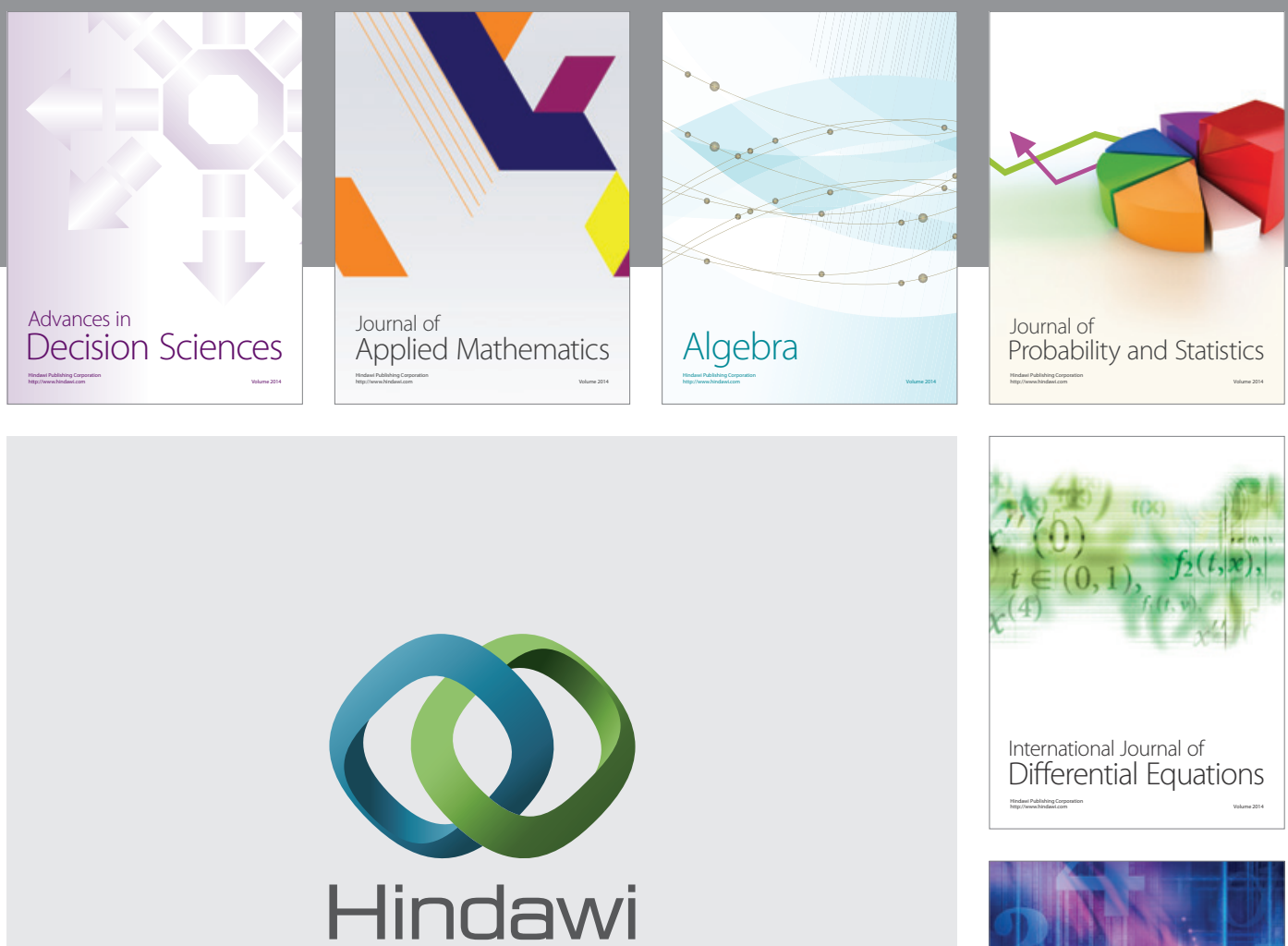

Submit your manuscripts at http://www.hindawi.com
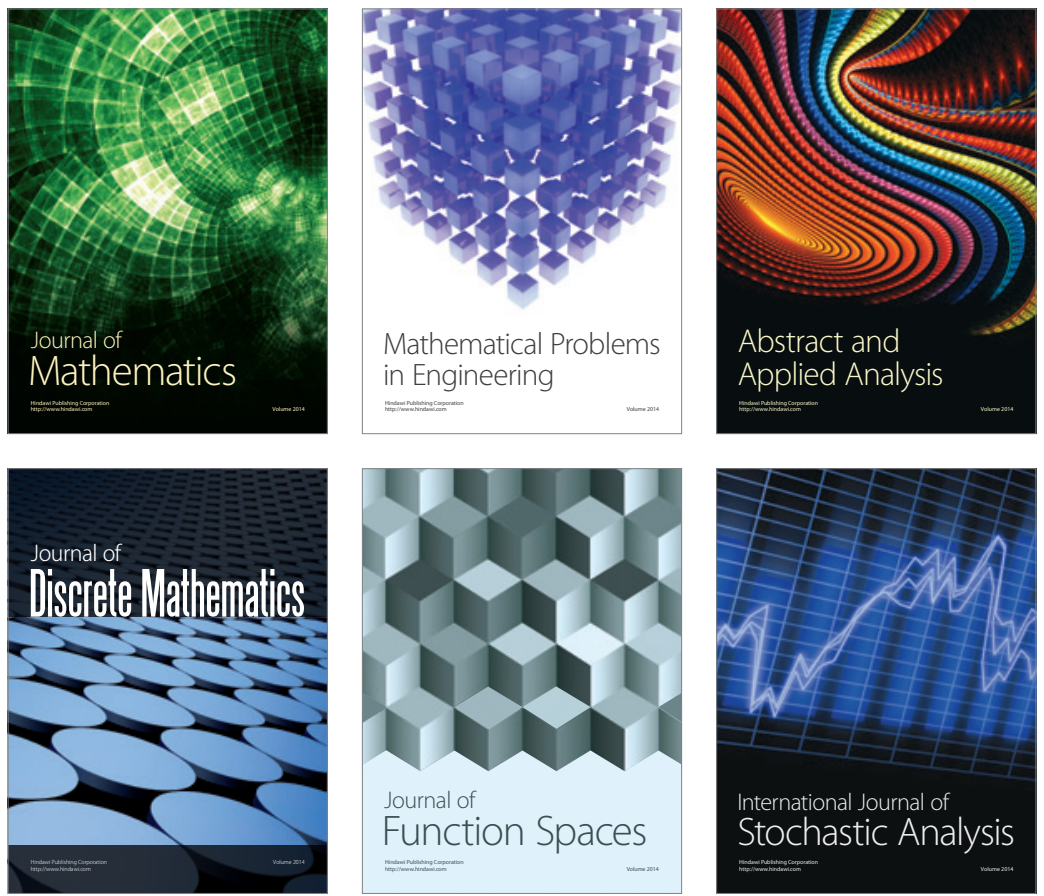

Journal of

Function Spaces

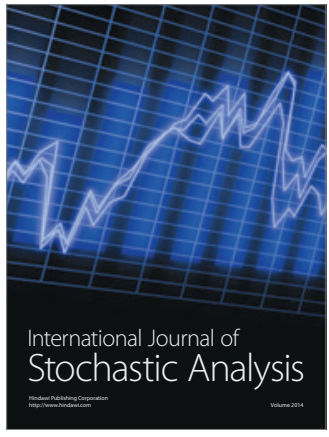

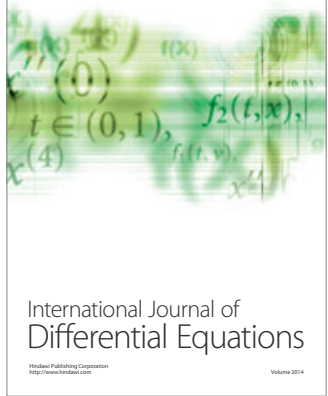
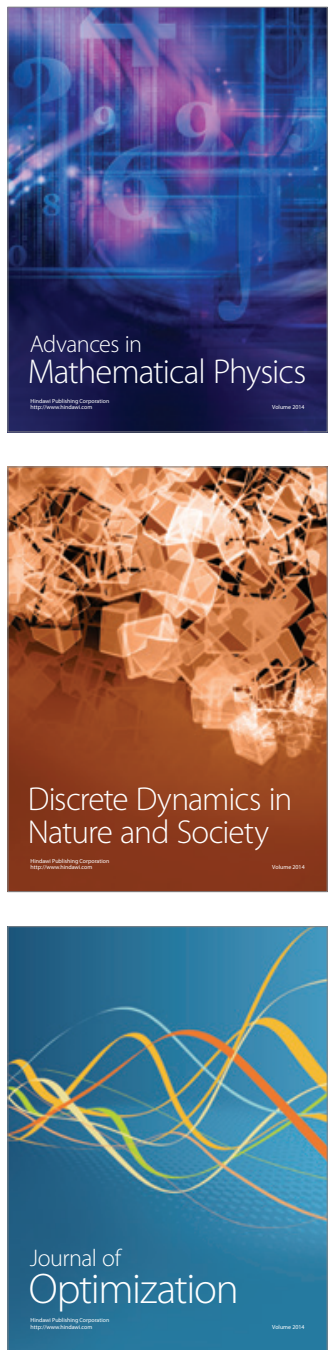